\title{
Chemopreventive Role of Preferential COX-2 Inhibitor Diclofenac in 9, 10- Dimethybenz(a)anthracene Induced Experimental Lung Carcinogenesis
}

\author{
Poonam Thakur and S.N. Sanyal*
}

Department Of Biophysics, Panjab University, Chandigarh-160014, India

* Corresponding Author:

Dr. S.N. Sanyal

Professor, Department of Biophysics

Panjab University, Chandigarh 160014,

India

Phone: +91-172-2534122

Email: sanyalpu@gmail.com

Received: 28 November 2009; | Revised: 4 March 2010; | Accepted: 26 April 2010

\begin{abstract}
Lung cancer was induced in female Wistar rats by a single intratracheal instillation of 9, 10Dimethybenz(a)anthracene (DMBA). To evaluate the chemopreventive potential of Diclofenac, which is a preferential cyclooxygenase-2 (COX-2) inhibitor, animals were divided into four groups. Group 1 received normal saline intratracheally, Group 2 DMBA $(20 \mathrm{mg} / \mathrm{kg})$, Group 3 a daily oral dose of Diclofenac $(8 \mathrm{mg} / \mathrm{kg})$ in addition to the DMBA while group 4 received Diclofenac alone. Animals were sacrificed after 6 and 12 weeks. Presence of tumors was confirmed by morphological and histological analysis. COX-2 expression was studied by immunohistochemistry (IHC) and Western immunoblot, which showed higher expression in DMBA treated animals but much lower in DMBA+Diclofenac. EtBr/AO co-staining of alveolar macrophages under fluorescence microscope showed that Diclofenac was able to induce apoptosis in DMBA treated animals. DNA fragmentation and TUNEL assay also showed similar results. To explore the apoptotic pathway further, Caspase-3 expression was studied by Western immunoblot which showed its absence in DMBA treated animals while present in the DMBA+Diclofenac group. Nitric oxide and citrulline levels were also quantitated which were lowered in the DMBA+Diclofenac group. The results indicate that Diclofenac might have beneficial effects in lung cancer chemoprevention and its action is mediated by COX2 inhibition and caspase- 3 activation.
\end{abstract}

Keywords: 9, 10- Dimethybenz(a)anthracene; intratracheal instillation; Diclofenac; lung cancer.

\section{Introduction}

Lung cancer is associated with highest number of cancer related deaths exceeding the mortality rate of breast, colon and prostate cancer combined [1]. The most common cause of developing lung cancer is cigarette smoking [2] which contains two main classes of carcinogensnitrosamines and polycyclic aromatic hydrocarbons $\quad$ (PAH) 
Dimethybenz(a)anthracene (DMBA) is a highly potent PAH which is widely used to induce a variety of cancers including that of the lung tissue [4,5 ]. To exert the carcinogenic effects it requires the metabolic activation by cytochrome P-450 enzyme (CYP1) [6] in a two step oxidation reaction. In the first step CYP1 B1 converts it into 7,12-DMBA-3,4-epoxide which is then further oxidized by microsomal epoxide hydrolase $(\mathrm{mEH})$ [7] to form 7,12-DMBA-3,4-diol. This is finally converted into its ultimate carcinogenic form 7,12DMBA-3,4-diol-1, 2-epoxide (DMBA-DE) by the action of CYP1 B1 again [8]. These metabolites form stable adducts with adenine and guanine residue in DNA leading to carcinogenesis [6].

Various chemopreventives have been tried in the past for intervention in lung cancer. However trials with natural agents such as retinoid, alphatocopherol and carotenoid etc. have not proved very successful and instead have shown adverse tumor promoting roles in the smokers [9]. Hence, lookout for alternative chemopreventive modalities is on. Many epidemiological studies have shown the efficacy of non steroidal anti inflammatory drugs (NSAIDs) in inhibiting a variety of cancers including colon, skin, bladder etc [10]. They act by inhibition of COX-2 enzyme which is seen to be upregulated in various malignancies including lung cancer [11] which is associated with poor prognosis [12]. It is over expressed in premalignant adenomatous hyperplasia, malignant adenocarcinoma and squamous cell carcinoma in lungs [13]. COX-2 has varied tumor promoting roles in lung cancer like angiogenesis, resistance to apoptosis, increased invasiveness, immunosupression, chronic inflammation etc. [14]. There is also evidence that PAHs in tobacco smoke can be converted into mutagens by $\mathrm{COX}-2$, utilizing its peroxidase activity [15].

Diclofenac is a preferential COX-2 inhibitor [16] and presents lesser side effects compared to the selective COX-2 inhibitors such as Rofecoxib and Valdecoxib whose use have been associated with cardiovascular risk and renal failure [14]. Hence, the present study was designed to examine the chemopreventive efficacy of Diclofenac in experimental lung carcinogenesis with emphasis on COX-2 expression and induction of apoptosis.

\section{Methods}

\subsection{Experimental design}

Female Wistar rats (200-220 g) were obtained from the Central Animal House of Panjab University, Chandigarh. Animals were kept in plastic cages under standard laboratory conditions and received food and water ad libitum. All the procedures were performed according to the conditions laid down by the ethics committee on the use of experimental animals of Panjab University. Animals were acclimatized for 1 week and then randomly assorted into 4 groups having 9 animals each. First group served as control and was given $0.5 \mathrm{ml}$ saline intratracheally, which is the vehicle of DMBA. Second group received a single dose of DMBA (20 mg/kg body weight) intratracheally. Third group was given Diclofenac ( $8 \mathrm{mg} / \mathrm{kg}$ body weight) dissolved in $0.2 \% \mathrm{CMC}$ (carboxy-methyl cellulose sodium salt) orally [17] in addition to DMBA. Fourth group received Diclofenac alone orally. DMBA was purchased from Sigma-Aldrich (St. Louis, MO, USA). The method for intratracheal instillation under ketamine anesthesia had been described by us earlier [18]. Animals were sacrificed after 6 and 12 week of dosing.

\subsection{Lung Tumor Analysis}

The animals were sacrificed by an overdose of diethyl ether at the indicated time interval after overnight fasting. The peritoneum was cut open and the heart perfused with chilled PBS. It cleared the lungs of all the blood. Then the lungs were removed and grossly examined for the presence of any surface tumors under a hand held lens and counted.

\subsection{Histology}

Lungs were removed, all five lobes separated and small portion of the tissue of about the same size were cut from two different lobes and fixed by immersing in $10 \%$ neutral buffered formalin. They were embedded in paraffin wax $\left(58^{\circ}-60^{\circ} \mathrm{C}\right)$ according to the standard technique [19] and cut into thin sections $(5 \mu \mathrm{M})$ by a hand operated microtome, and stained with hematoxylin and eosin. 


\subsection{Immunohistochemistry}

Paraffin embedded lung sections were dewaxed in xylene, hydrated and incubated with $2 \% \mathrm{BSA}$ in PBS for $30 \mathrm{~min}$ in a moist chamber to block non specific staining. After rinsing in PBS, they were incubated with primary antibody against COX-2 (1:1000 dilution) obtained from Santa Cruz Biotechnology Inc., CA (USA), with $1 \%$ BSA in PBS for 2 hours in moist chamber at $37^{\circ}$ C. The slides were washed twice with PBS-tween $(50 \mu \mathrm{l} / 100 \mathrm{ml}$ of PBS) and incubated with the secondary antibody (1:10,000 dilution) from Genei, Bangalore (India), in 1\% BSA for 2 hour at $37^{\circ} \mathrm{C}$. Washing with tween is repeated and slides incubated with BCIP/NBT (Genei, Bangalore (India)) solution in dark and color development monitored. Slides were counter stained with methyl green, dehydrated and mounted in DPX [20].

\subsection{TUNEL}

This assay was based on the identification of DNA nicks by terminal deoxynucleotidyl transferase, an enzyme that catalyzes the addition of dUTPs that are secondarily labeled with a marker. The procedure was carried out according to the instruction given in the kit by the manufacturer (Trevigen, USA).

\subsection{DNA fragmentation}

DNA was isolated from the lung tissue by phenol chloroform extraction method [21]. After quantitation at $260 \mathrm{~nm}$, the electrophoresis was carried out on $1.5 \%$ agarose gel in TAE buffer. Gel was visualized by ethidium bromide under UV light and photograph taken using a GelDoc.

\subsection{Fluorescence microscopy for apoptosis study in alveolar macrophages}

Animals were sacrificed and lungs perfused with chilled PBS. They were removed, washed in PBS and chopped finely. They were kept on rocker with protease for 1 hour, filtered through a muslin cloth and the filtrate centrifuged at 700 rpm for 5 min. Pellet obtained was suspended in PBS. Viability of the cells was checked by the methyl green staining and purity of preparation checked with Giemsa. The cell preparation was found to be more than $97 \%$ pure. $10 \mu \mathrm{l}$ of isolated cells were mixed with equal volume of both Acridine orange and Ethidium bromide or Propidium iodide and Hoest-33342 [22]. Cover slip was put on slide and observed under florescence microscope (Nikon, Japan). Live cells appear green, apoptotic cell yellow while necrotic cell red with Acridine orange-Ethidium bromide. For Propidium iodide-Hoest-33342, live cells appear blue, apoptotic cells pink and necrotic cells red [23]. Quantitation is done by counting a total of 100 cells from 4 different slides and percentage of the apoptotic cells calculated for each group.

\subsection{Western blotting}

It was done for COX-2 and Caspase-3. Protein was extracted using the protocol based on Sambrook et al [21] and estimated by Bradford method [24]. Protein was separated on SDSPAGE, transferred onto the nitrocellulose membrane and blotting done by the method of Towbin et al [25] using the specific primary antibody as above. Blots were developed using NBT/BCIP solution and photographed.

\subsection{Nitric oxide synthase (NOS) assay}

It was estimated indirectly by quantitating the levels of nitric oxide (NO) and L-citrulline which are produced by the action of NOS. NO quantitation was done using the protocol of Stuehr and Marletta [26] by Griess reagent which gives pink color on reaction with the stable product nitrite and can be read at $540 \mathrm{~nm}$. For citrulline estimation the method adopted was that of Boyde and Rahmatuleen [27]. The assay was based on its reaction with diacetylmonoxime and absorbance measured at $530 \mathrm{~nm}$.

\subsection{Statistical Analysis}

Statistical analysis of the data was performed by the analysis of variance (one way ANOVA) following Post-Hoc test using the least significance difference (LSD) method with the help of a SPSS v 10 computer software.

\section{Results}

No morphological changes on the lung surface were seen at 6 week of DMBA treatment. However, after 12 week tumor nodules were 
observed in all the DMBA treated animals (Fig. 1). In addition, angiogenic lesions were also seen along with tumors. About $33 \%$ of the animals in DMBA+Diclofenac group had lesions in lungs but no tumorous nodules observed. The tumor incidence, burden and multiplicity are shown in table 1, which revealed considerable improvement in these parameters after Diclofenac treatment.

Table 1- Chemopreventive response of Diclofenac on the DMBA induced lung carcinogenesis in rats in terms of reduction in tumor and lesion incidence.

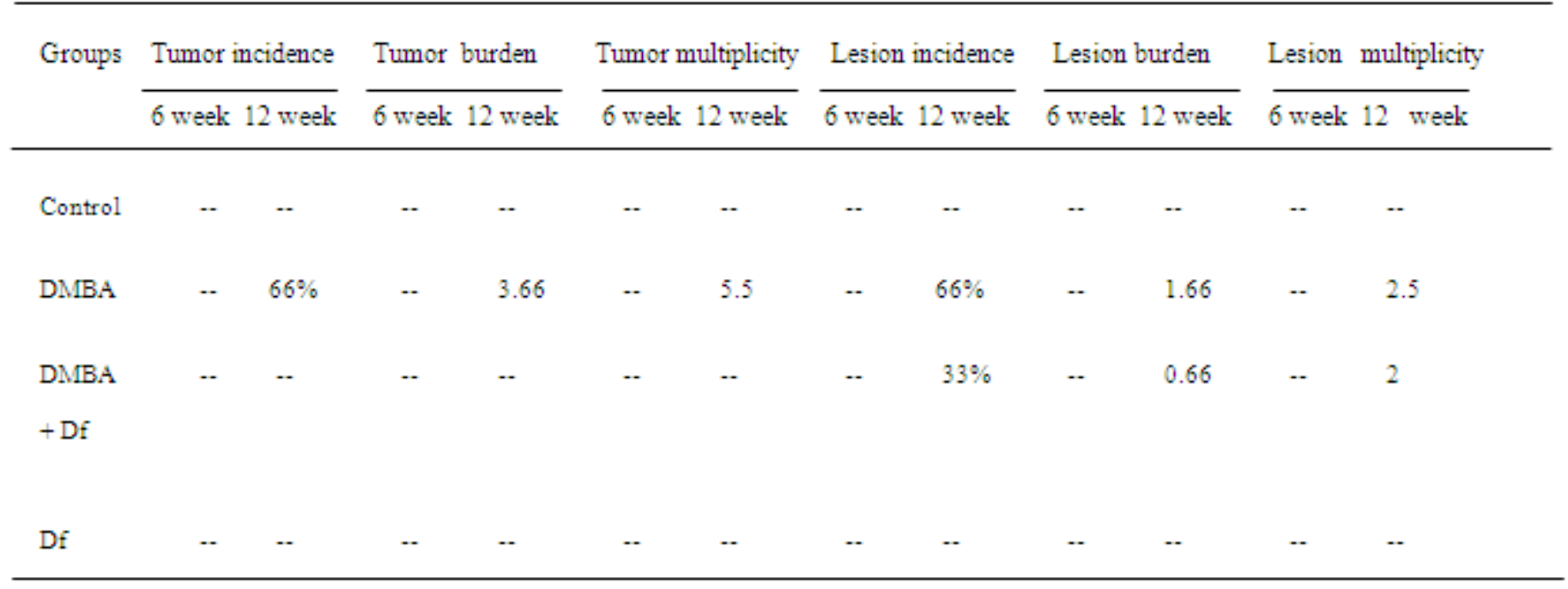

Df- Diclofenac; DMBA-9, 10- Dimethybenz(a)anthracene

Incidence: percentage of animals bearing tumor or lesion.

Burden: total no of tumors or lesions/total no of rats.

Multiplicity: total no of tumors or lesions /no of tumor or lesion bearing rats.

Histopathological examination of lung sections was done. At 6 week, although no tumors were observed on the surface the DMBA treated group showed mild dysplasia and alveolar hyperplasia (Fig. 2-B), while alveoli were intact. As can be seen in fig. 2-F, at 12 week adenoma with alveolar dysplasia was present marked by dense cellular proliferation in the regions adjoining adenoma. The lesion present in DMBA+Diclofenac showed some altered histology as the alveolar spaces were constricted compared to the control group. Hyperplasia was also seen. The animals treated with Diclofenac alone showed no histopathological alteration.

COX-2 expression when observed by IHC showed higher expression in DMBA treated animals both at 6 week (Fig. 3-B) and at 12 week (Fig. 3-F). At 6 week marginal expression was also seen in DMBA+Diclofenac group while at 12 week the expression was confined mostly to the constricted alveoli. Control as well as Diclofenac only groups showed negligible expression of COX-2. Western blot analysis also showed higher expression of COX-2 in DMBA treated group which was lowered in DMBA+Diclofenac group (Fig. 4-A).

DNA fragmentation study characteristic of apoptosis in Fig. 5 shows the absence of apoptosis in DMBA treated animals while the bands of fragmented DNA were seen to be induced by Diclofenac treatment in group 3. The bands were however, absent in Diclofenac only group. TUNEL assay showed apoptotic cells in brown stain which were then counted and expressed as the percent apoptotic cells. Fig. 6 shows the lowered number of apoptotic cells in DMBA treatment compared to the control group at 6 week $(\mathrm{p} \leq 0.001)$ which was also further diminished at 12 week $(\mathrm{p} \leq 0.01)$. Diclofenac treatment was able to restore apoptosis at both the time points. 

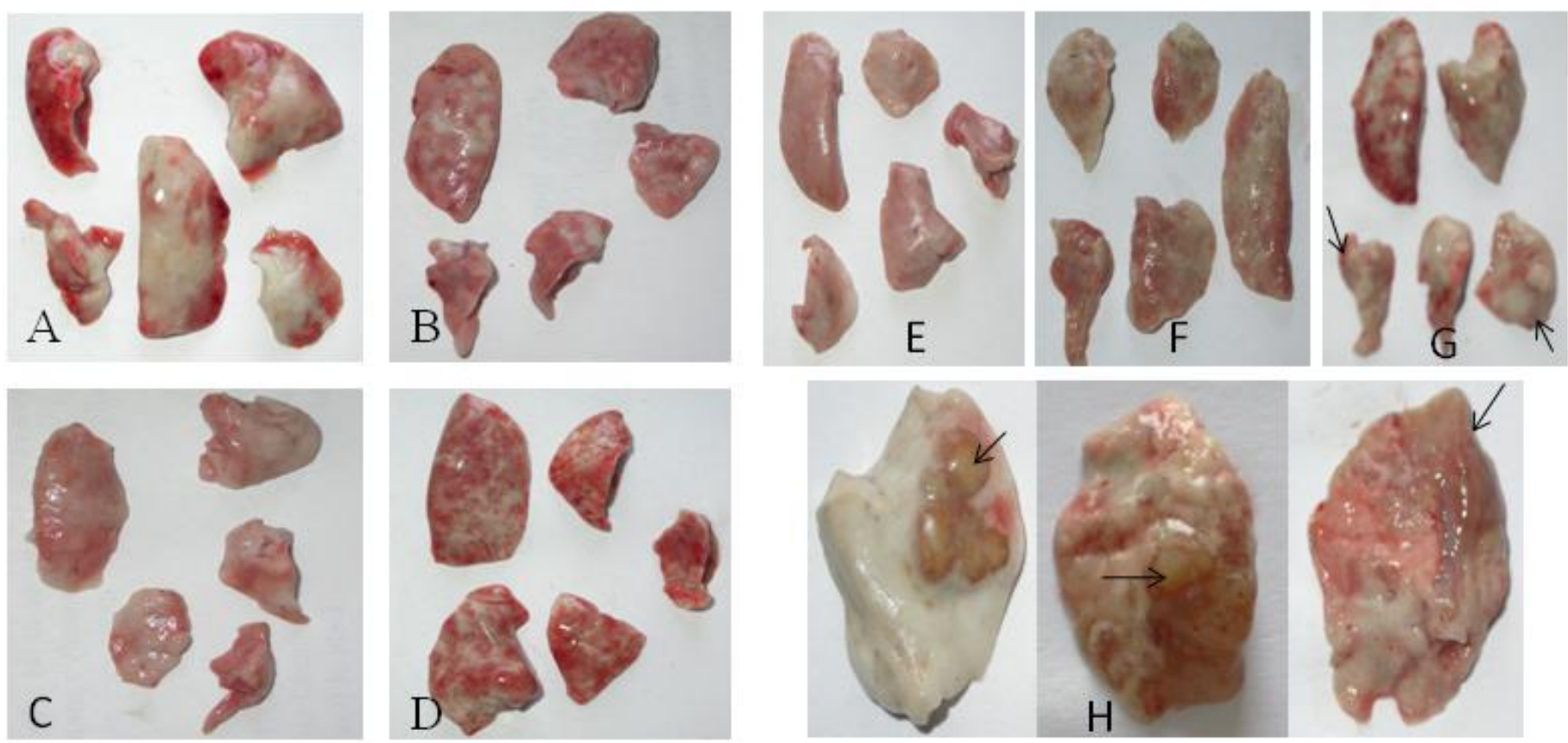

Figure 1. Effects of DMBA, DMBA+ Diclofenac and Diclofenac only treatment on the gross anatomical changes in the rat lungs. A-Control, B-DMBA, C-DMBA+Diclofenac and D-Diclofenac treatment groups for 6 week. No tumors were observed in any of the groups. While E-control, F-DMBA, G-DMBA+ Diclofenac and H-Diclofenac treated lungs for 12 week. Visible tumor nodules and lesions in DMBA treated animals are marked by arrows.
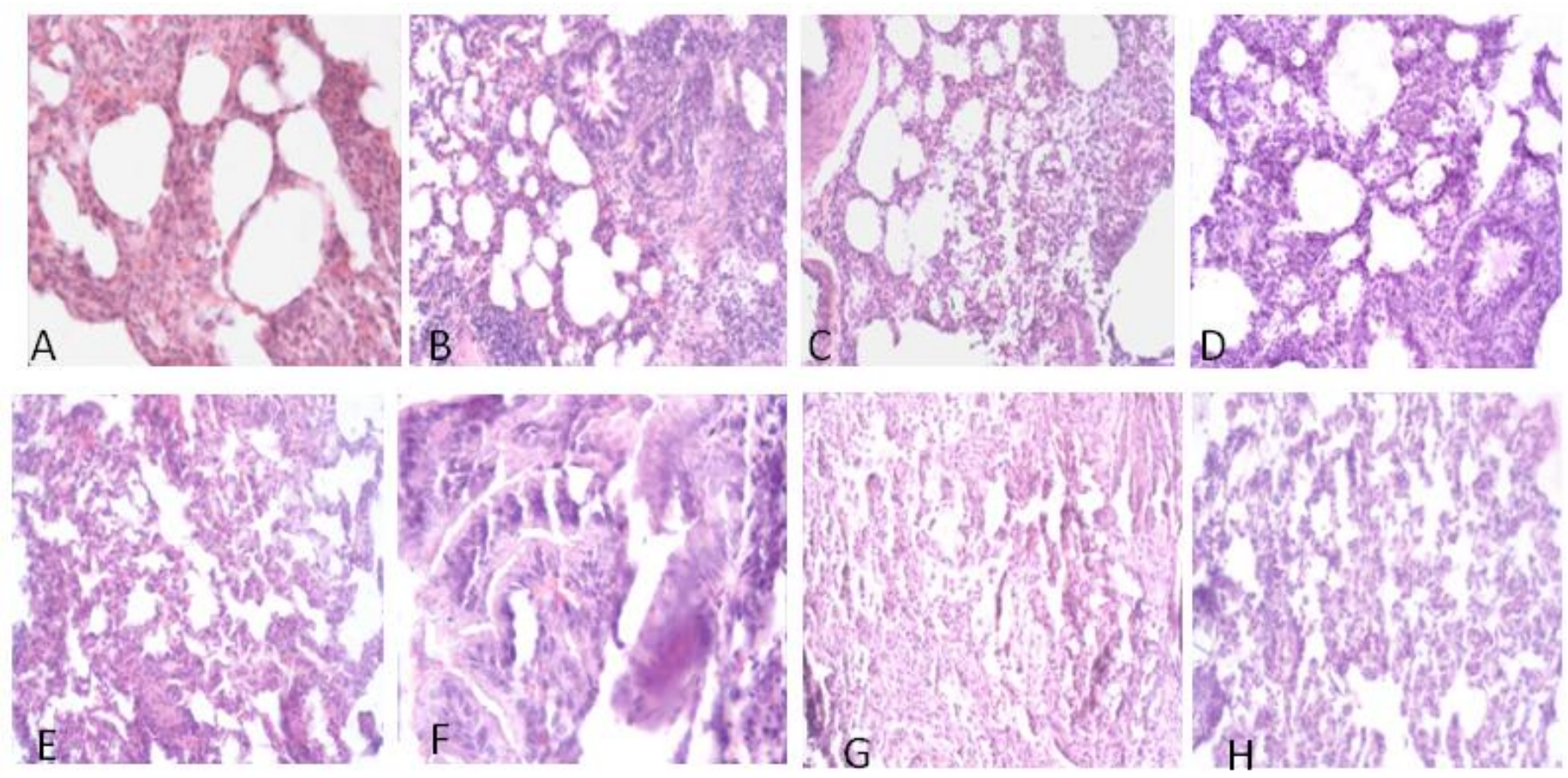

Figure 2. Histoarchitectural changes seen in rat lungs by Haematoxylin-Eosin staining. Top panel shows the AControl(40X), B-DMBA(20X), C-DMBA+Diclofenac(20X) and D-Diclofenac(20X) treatment groups for 6 week. Mild dysplasia can be seen in DMBA treated group. In the bottom panel E-Control(20X), F-DMBA(40X), G- 
DMBA+Diclofenac(20X) and H-Diclofenac(20X) for 12 week of treatment. Adenoma along with cellular proliferation in adjoining region is visible in DMBA group.
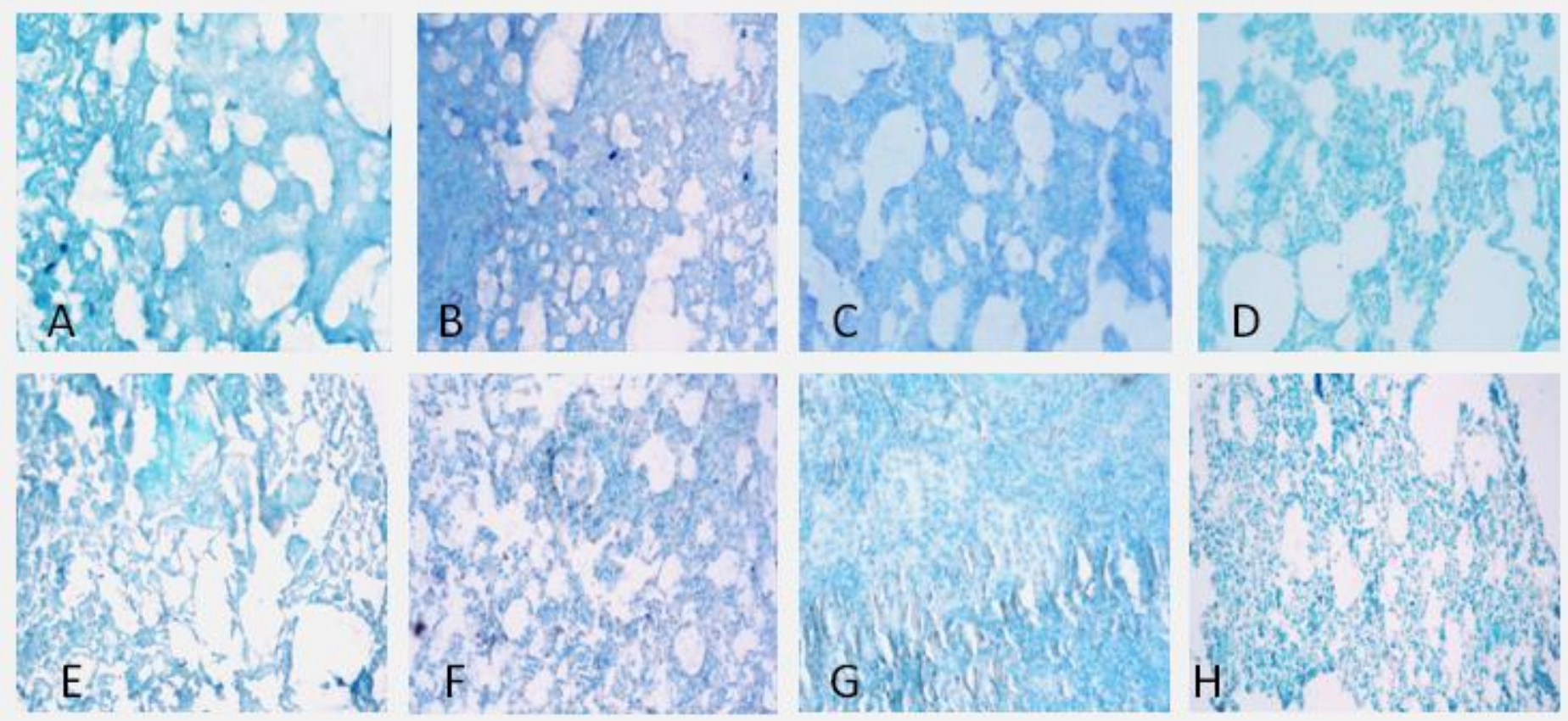

Figure 3. The expression of COX-2 in the rat lungs seen by immunohistochemistry. Cells were stained with methyl green while COX-2 expression was seen as blue color. A-Control, B-DMBA, C-DMBA+Diclofenac and D-Diclofenac treatment groups for 6 week. E-Control, F-DMBA, G-DMBA+Diclofenac and H-Diclofenac treatment groups for 12 week. 20X. Higher expression of COX-2 can be seen in B and F, which was mostly cytoplasmic.

A. $\mathbf{C O X}-2$

B. Caspase-3

\section{Control DMBA DMBA Diclofenac} +Diclofenac

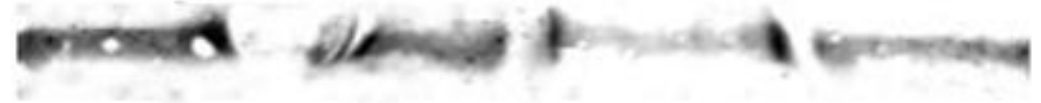

6 week

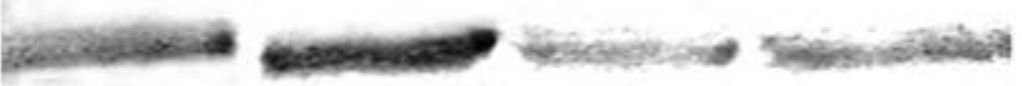

12 week

6 week

12 week
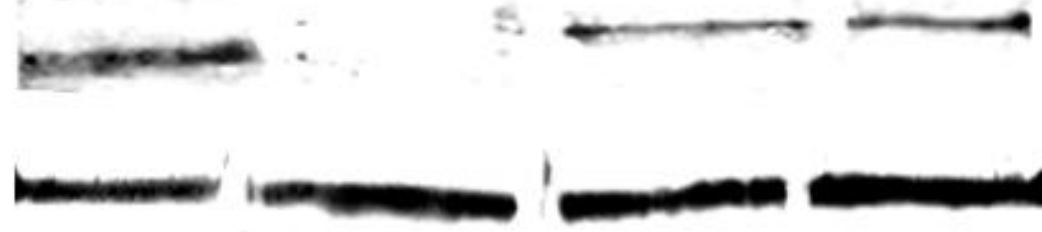

I 
Figure 4. The immunoblot analysis of (A) COX-2 and (B) Caspase-3 in rat lung after Diclofenac treatment.

Fig. 4-B shows the Caspase-3 expression as seen by the western immunoblot which was negligible in DMBA treatment. However, with Diclofenac co-administration the expression was restored back to normal level similar to control group. Since alveolar macrophages are involved in inflammation and play an important role in cancer progression, apoptosis was studied by staining with fluorescent dyes PI/Hoest-33342 and EtBr/AO, and observed under fluorescent microscope. Fig. 7 shows the percent apoptotic cells in the different groups at 6 week. Both PI/Hoest-33342 and EtBr/AO showed the similar trend. The number of apoptotic macrophages had drastically been reduced $(\mathrm{p} \leq 0.001)$ in the 12 week DMBA treatment (Fig. 8) and to a lesser extent at 6 week. At early stages, the restoration of apoptosis by Diclofenac was near the control value but with the progression of the tumor the restoration was not complete and the number of apoptotic cell was significantly $(p<0.001)$ lower than the control, although the number was higher compared to the DMBA group $(p \leq 0.001)$. Diclofenac only group also had apoptotic cell count lower than the control group.
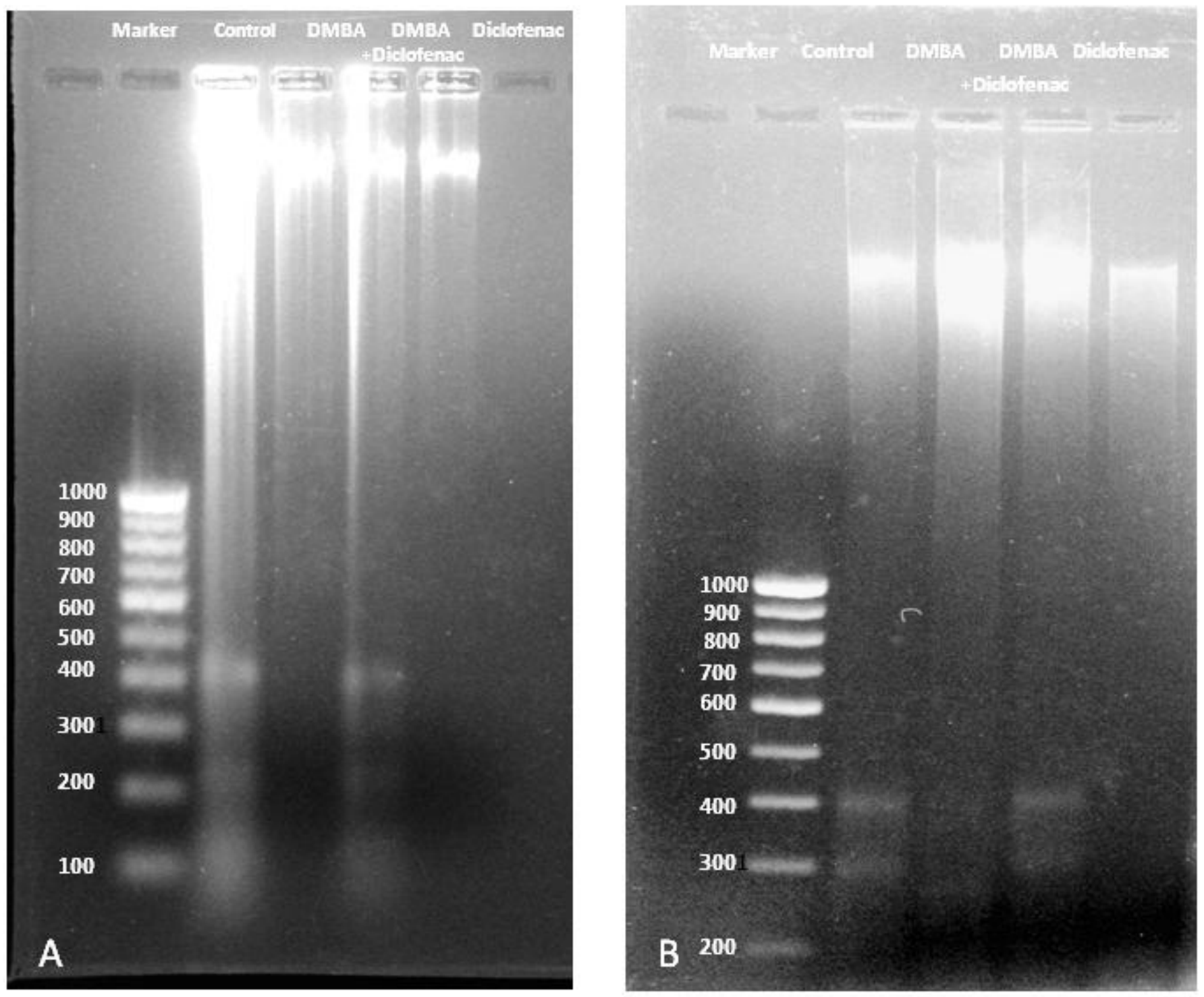

Figure 5. Apoptosis as seen by DNA fragmentation for (A) 6 week and (B) 12 week of treatment. 

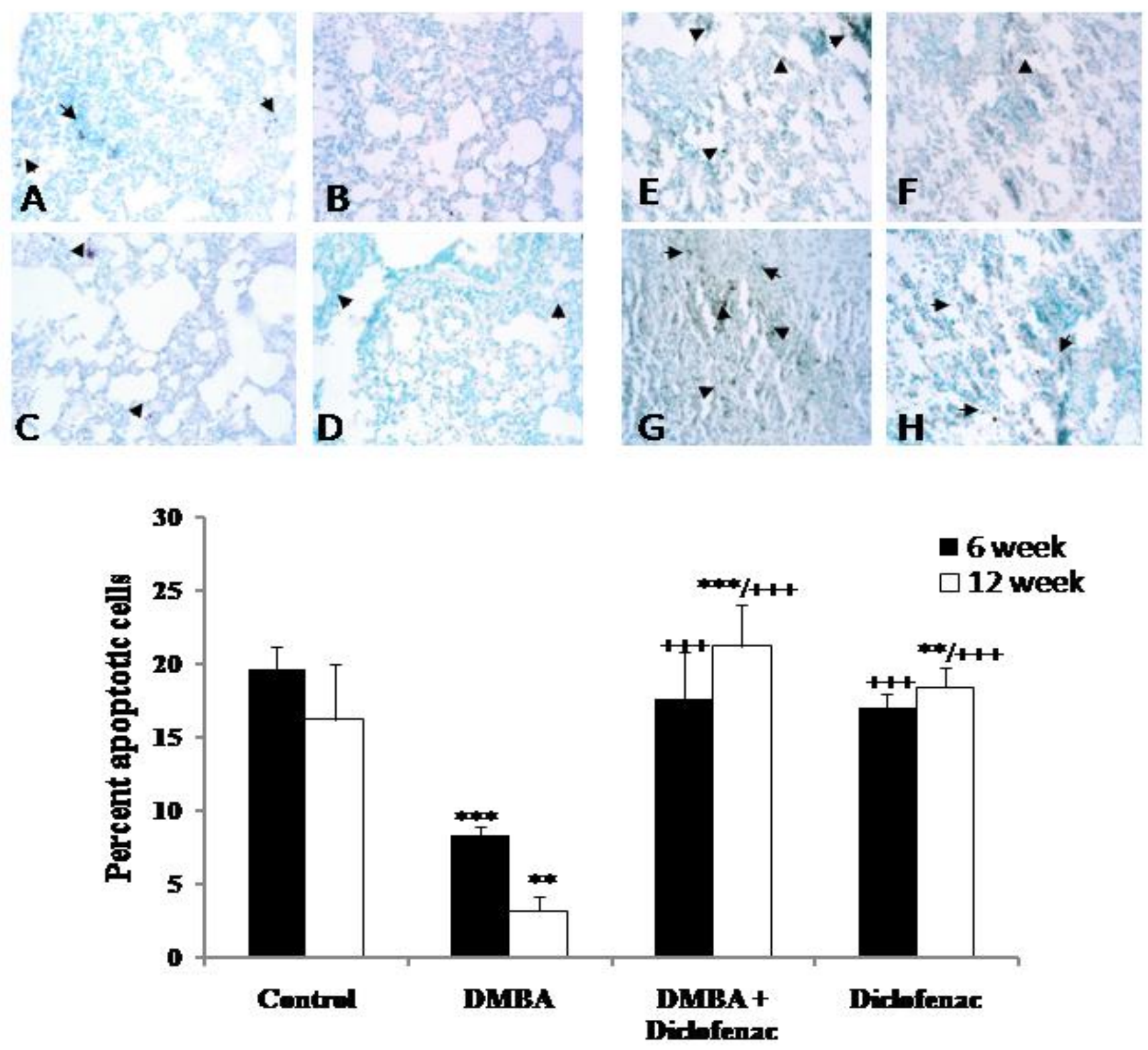

Figure 6. TUNEL assay in lung sections in various treatment groups. TUNEL positive cells are seen in brown color while rests of the cells appear cyan with methyl green stain. A-Control, B-DMBA, C-DMBA+Diclofenac and DDiclofenac treatment groups for 6 week. E-Control, F-DMBA, G-DMBA+Diclofenac and H-Diclofenac treatment groups for 12 week. 20X. Histogram shows the percent apoptotic TUNEL positive cells in groups $(* * * p \leq 0.001$ w.r.t. control group, $* * \mathrm{p} \leq 0.01$ w.r.t. control group, $+++\mathrm{p} \leq 0.001$ w.r.t. DMBA group).

iNOS is produced by macrophages and known to be upregulated during carcinogenesis. Fig. 9-A show that NO levels were lowered in DMBA group when compared to control $(\mathrm{p} \leq 0.01)$ at 6 week but increased at 12 week. Diclofenac treated animals in DMBA+Diclofenac group and Diclofenac only group showed a significantly higher level $(\mathrm{p} \leq 0.001)$ of NO compared to control at 6 week but the level was reduced at 12 week. Citrulline levels also showed the trend similar to NO level (Fig. 9-B).

\section{Discussion}

(C) 2010 by NWPII. All rights reserved. 
In the present study a single intratracheal instillation had been employed to produce lung tumors in female Wistar rats. Various other protocols for induction of lung cancer are in use which includes inhalation of tobacco smoke [28], injections of benzopyrene [29], 4(methylnitrosamino)-1-(3-pyridyl)-1-butanone [30] etc. Compared to these methods, the current method has advantage that a single dose is able to induce adenocarcinomas in comparatively lesser time. It allows the carcinogen to be delivered accurately to the lung tissue only, thus overcoming the possibility that the cancer might have developed secondarily by metastasis from other organs as can happen when the carcinogen is given intraperitonialy or other routes.
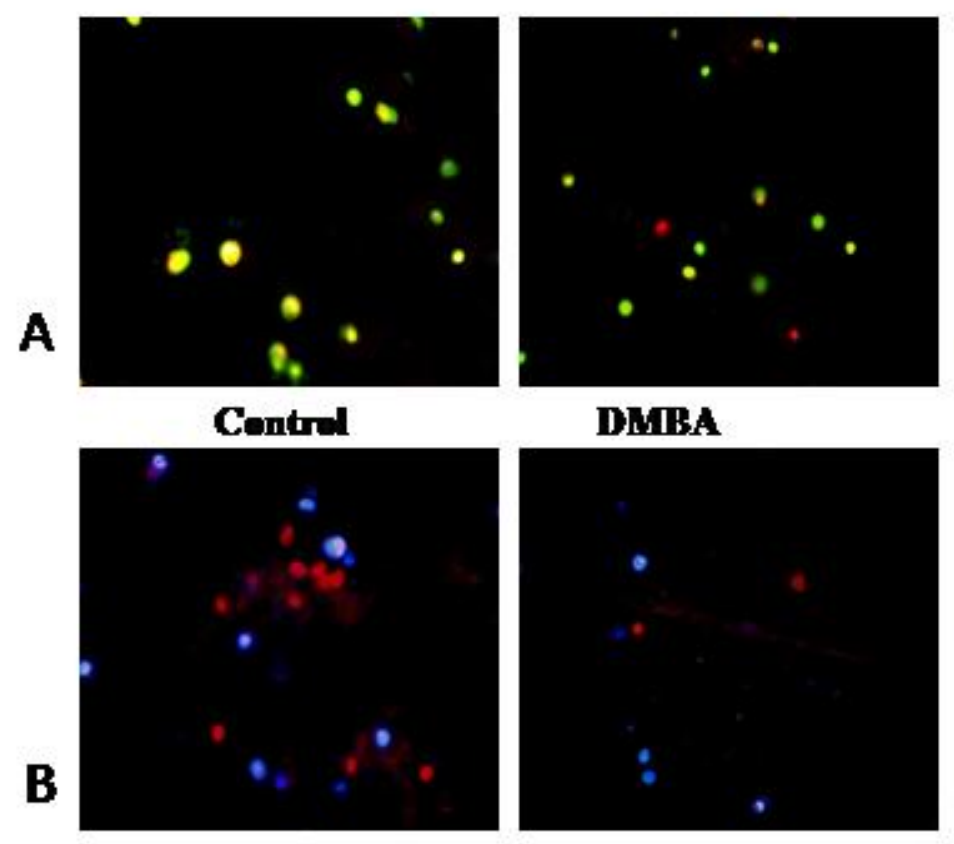

DMBA
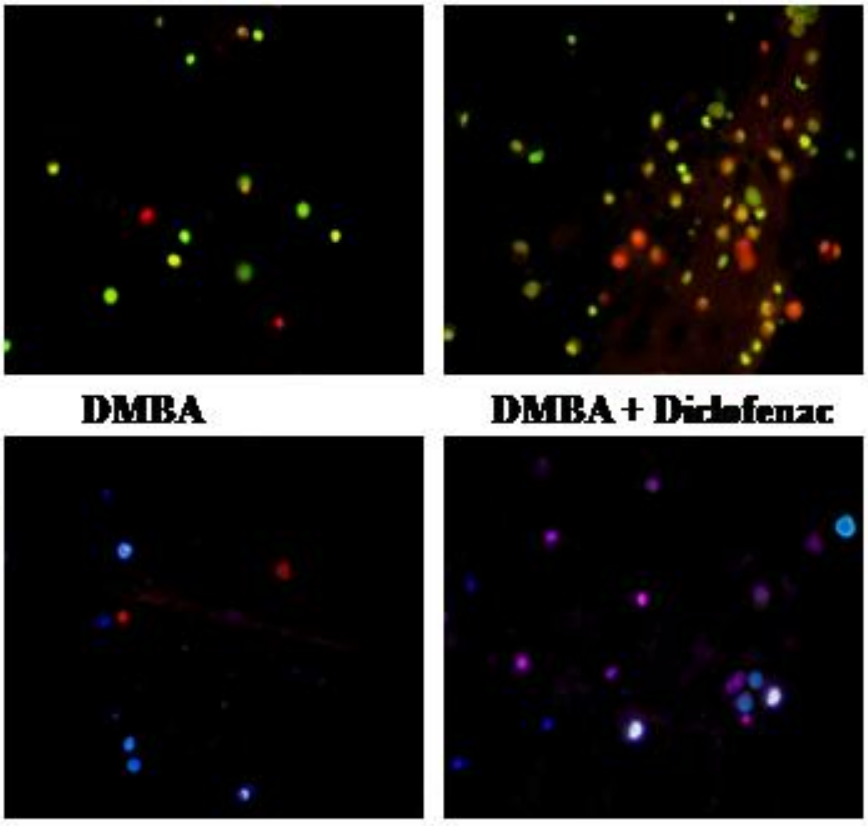

DMBA + Didefenac
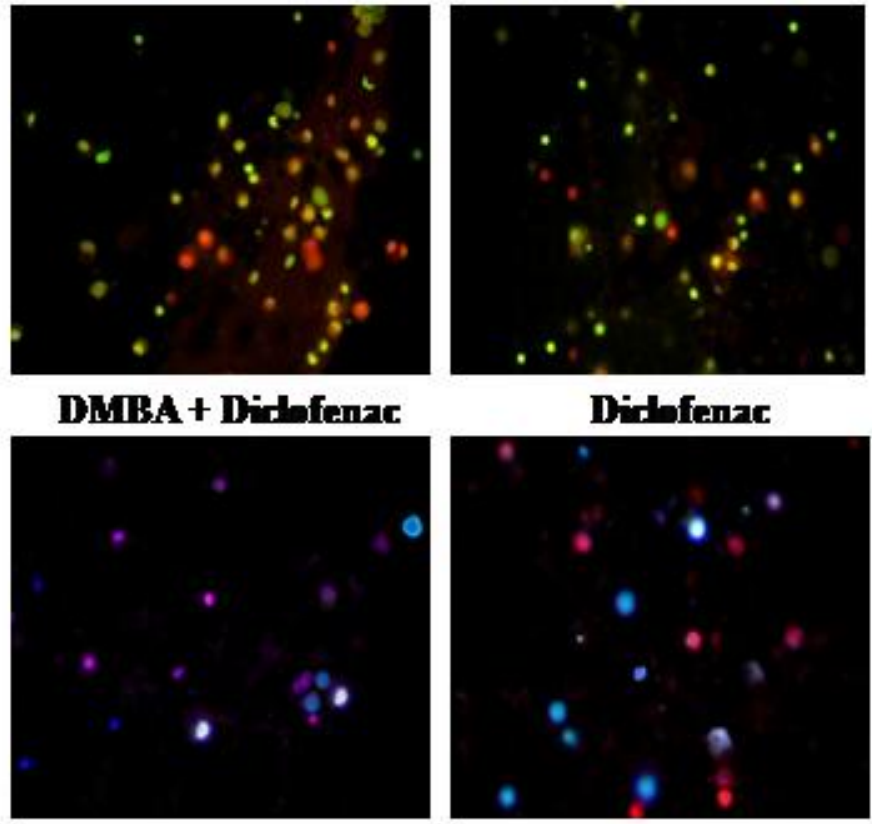

Dirlofenac

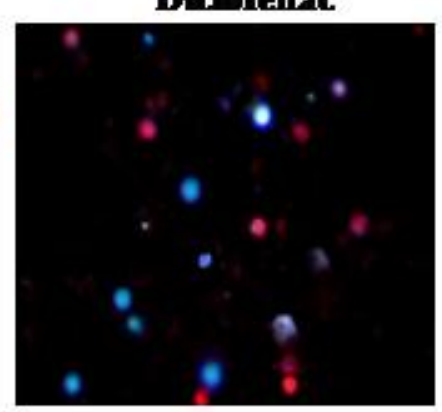

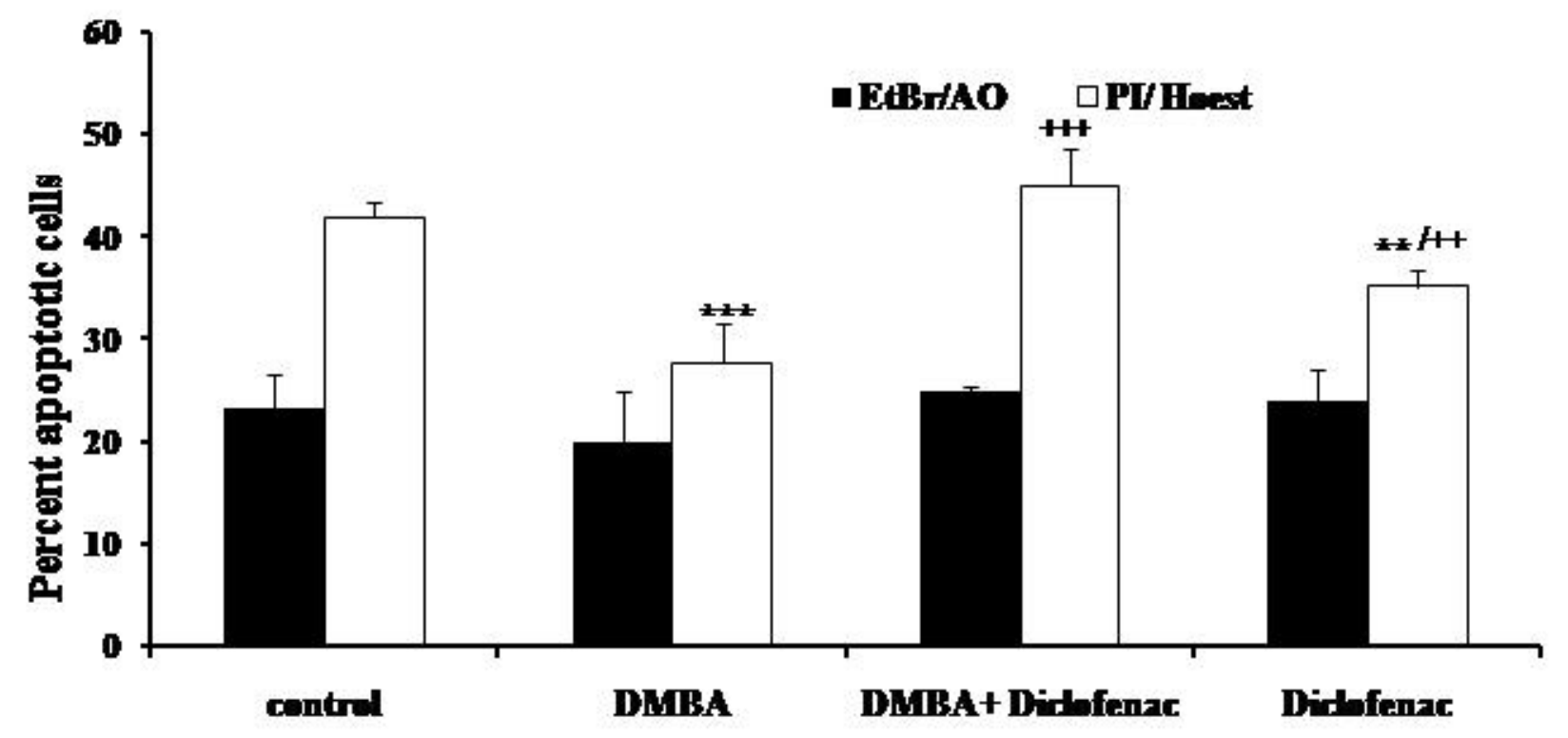

Figure 7. Macrophages visualized under fluorescence microscope (20X) for 6 week treatment group. (A) Ethidium Bromide/Acridine Orange co-staining- Apoptotic cells appear yellow, viable cells are green while necrotic cells appear as red colored. (B) Propidium iodide /Hoechst 33342 co-staining: Pink colored cells are apoptotic; viable 
cells appear blue and necrotic cells red. Histogram shows the percent apoptotic cells $(* * * p \leq 0.001$ w.r.t. control group, ${ }^{* *} \mathrm{p} \leq 0.01$ w.r.t. control group, $+++\mathrm{p} \leq 0.001$ w.r.t. DMBA group, $+\mathrm{p} \leq 0.05$ w.r.t. DMBA group).
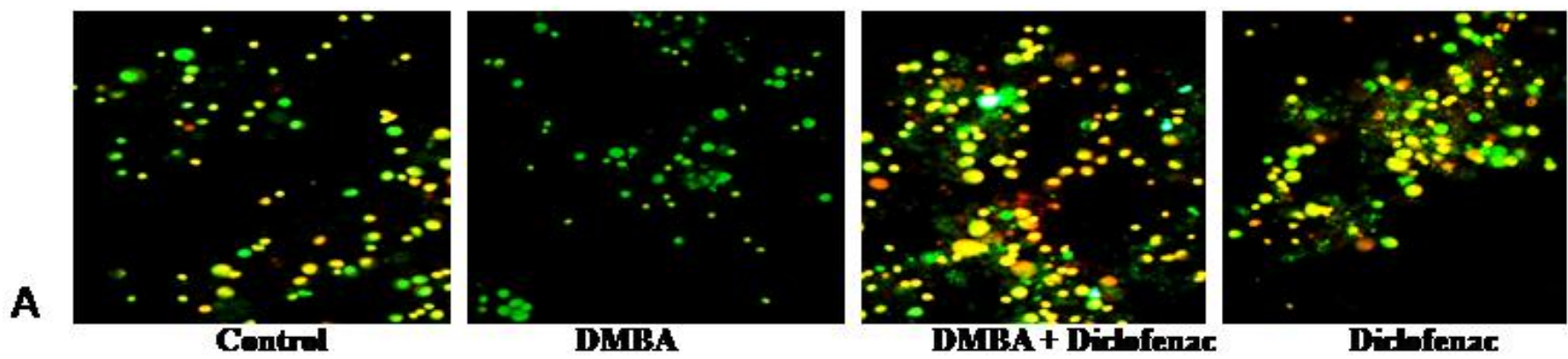

Didfenac
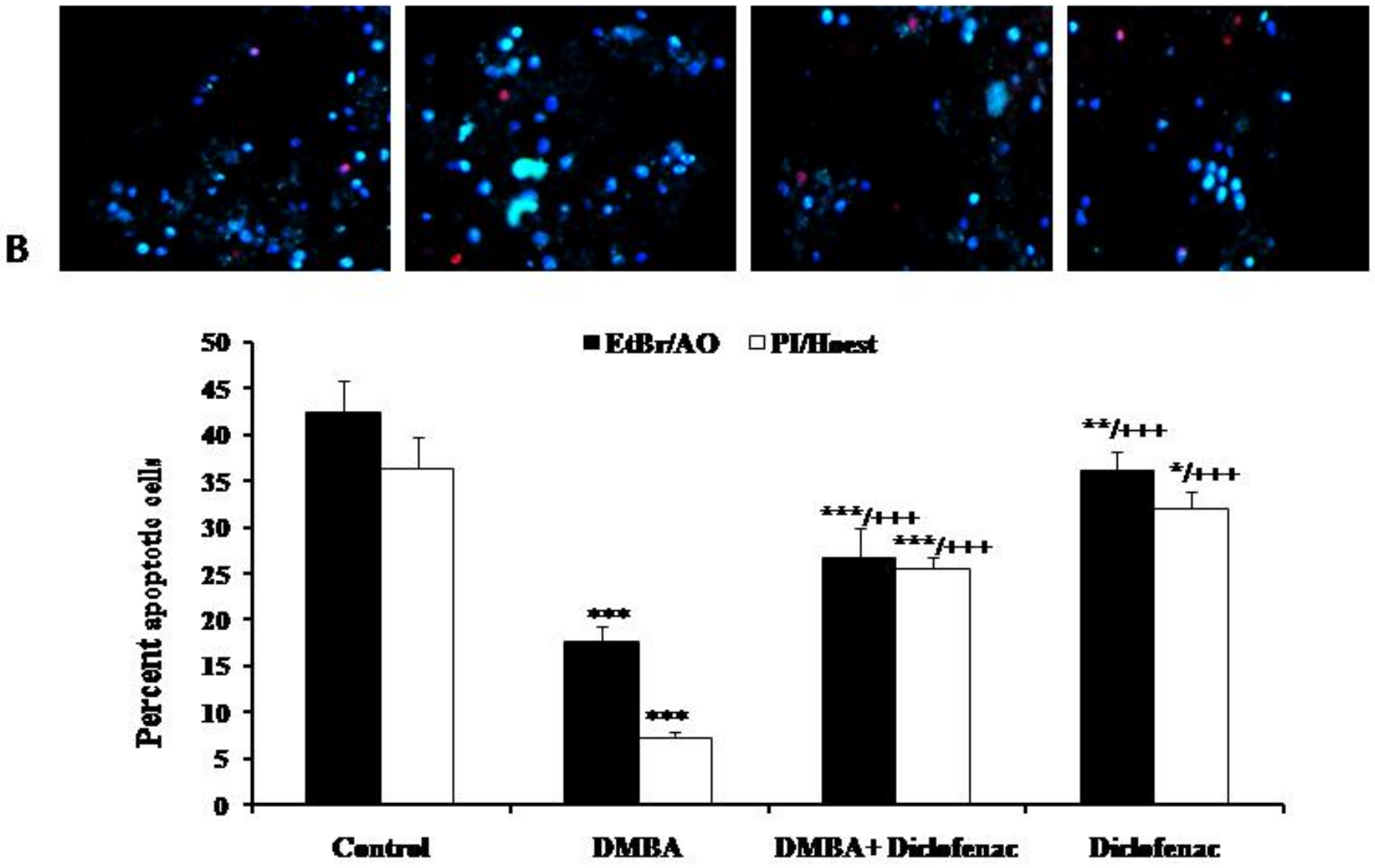

Figure 8. Macrophages visualized under fluorescence microscope (20X) for 12 week treatment group. (A) Ethidium Bromide/Acridine Orange co-staining. (B) Propidium iodide/Hoechst 33342 co-staining. Histogram shows the percent apoptotic cells $\left({ }^{* * *} \mathrm{p} \leq 0.001\right.$ w.r.t. control group, ${ }^{* *} \mathrm{p} \leq 0.01$ w.r.t. control group, ${ }^{*} \mathrm{p} \leq 0.05,+++\mathrm{p} \leq 0.001$ w.r.t. DMBA group).

DMBA has been widely used for inducing a variety of cancers including mammary, skin and lung cancer [4]. DMBA, like other polycyclic aromatic hydrocarbons (PAHs), need metabolic activation for carcinogenic effect [31]. Thus tumor development is a time dependent event, and tumor incidence and tumor burden increase with time. Diclofenac caused the prevention in cancer Am. J. Biomed. Sci. 2010, 2(3), 275-288; doi: 10.5099/aj100300275 incidence as evidenced by the absence of any tumor nodules and able to prevent the occurrence of lesions to a large extent. This is consistent with the reports suggesting that NSAIDs may indeed be the effective anticancer agents [32].

Role of COX-2 in inflammation has been well elucidated. Also, it is an immediate early response gene product and progressively upregulated during (C) 2010 by NWPII. All rights reserved. 
carcinogenesis [33]. Diclofenac exerts its chemopreventive action by the inhibition of COX2 , which is unique among the NSAIDs as having a preferential selectivity for COX-2 in vivo [34]. It has a lower $\mathrm{IC}_{\mathbf{5 0}}$ value than other nonselective NSAIDs [16] and hence, has a better gastrointestinal safety profile than the traditional NSAIDs [35]. There has also been an upthrust in the use of COX-2 selective NSAIDs as chemopreventives in recent times but the reports of renal failure and cardiovascular complications [36] have forced the researchers to use them with caution. The present results suggest that Diclofenac's chemopreventive action is mediated by the inhibition of COX-2 as seen by IHC as well as western blot where DMBA and Diclofenac coadministration leads to a lowering of the enzyme levels.
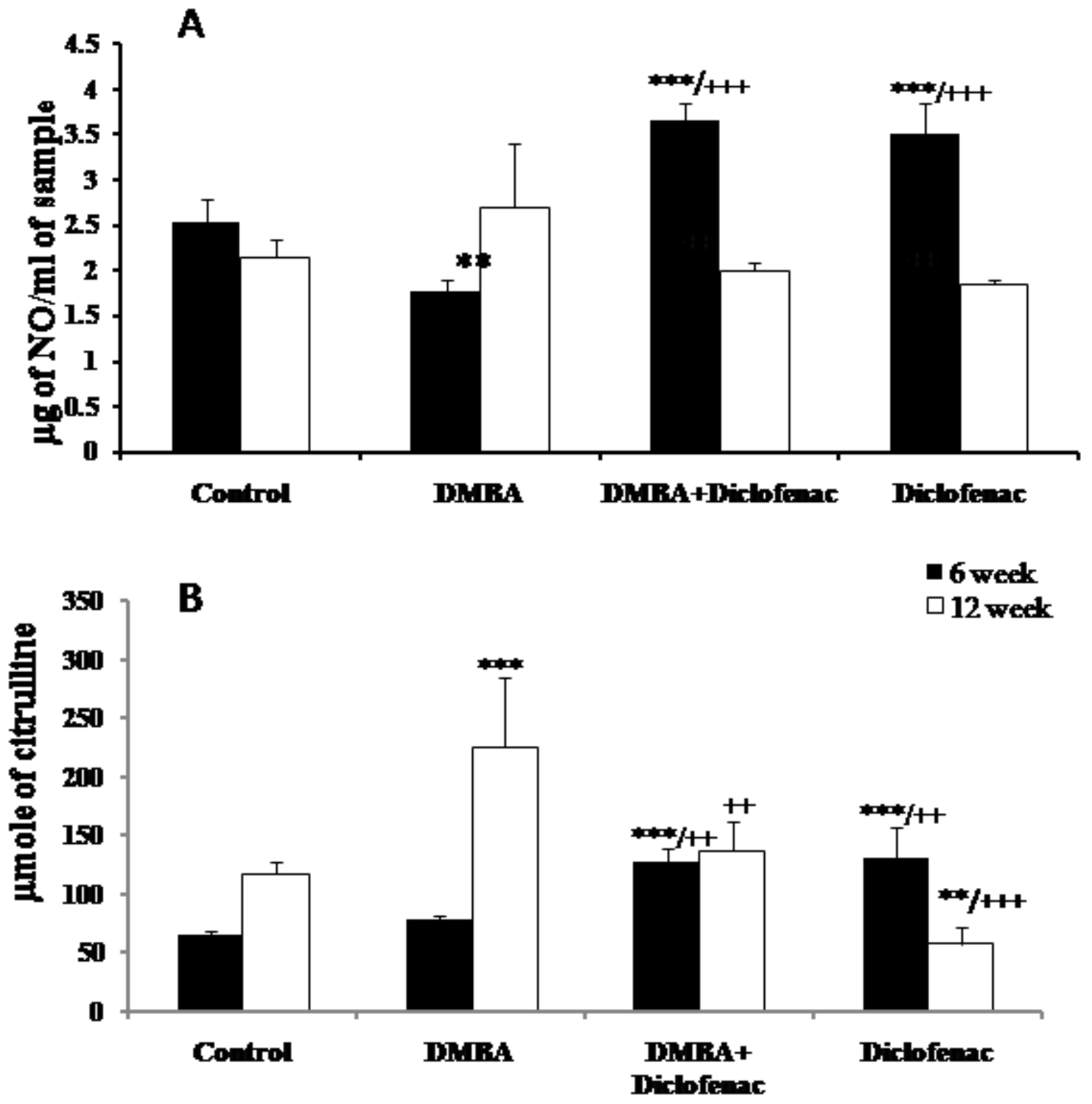

Figure 9. (A) Histogram showing the variation in nitric oxide level in various treatment groups at 6 week and 12 week $(* * * \mathrm{p} \leq 0.001$ w.r.t. control group, $* * \mathrm{p} \leq 0.01$ w.r.t. control group, $+++\mathrm{p} \leq 0.001$ w.r.t. DMBA group, $+\mathrm{p} \leq 0.05$ w.r.t. DMBA group). (B) Histogram showing the variation in citrulline level in various treatment groups at 6 week and 12 week $(* * * \mathrm{p} \leq 0.001$ w.r.t. control group, $* * \mathrm{p} \leq 0.01$ w.r.t. control group, $+++\mathrm{p} \leq 0.001$ w.r.t. DMBA group, $++\mathrm{p} \leq 0.01$ w.r.t. DMBA group). 
Diclofenac evidently exerts its end effect by the induction of apoptosis in cancerous tissue [37], as also seen in the present study, probably by an activation of caspase-3. Apoptosis is dysregulated during carcinogenesis [38] and its restoration is believed to be mediated by activation of caspase- 3 [39] which is the major protease in apoptotic pathway. Interestingly, caspase-3 levels were decreased at 6 week in DMBA treated animals even before the tumors were evident. DNA fragmentation also showed that even though tumors were not present initially, the apoptosis pathway has been disrupted and lead to the reduction of apoptosis. Similar results were also seen in TUNEL assay where Diclofenac treatment restored the apoptosis which might be due to the activation of caspase- 3 as evidenced by western blot results.

DMBA, like other polycyclic aromatic hydrocarbons, generates inflammation during carcinogenesis [4] and may attract the lung derived immune cells at the tumor site. Alveolar macrophages are involved in immune response and inflammation in lung, which may play an important role in the regulation of tumor growth. Also, tumor associated macrophages are activated to secrete large number of growth factors, cytokines, inflammatory mediators and proteases. These effects favor tumor metastasis and invasiveness [40]. The lowering of apoptosis in macrophages as evident by fluorescent staining in the present study suggests that they play a key role in tumor formation. These macrophages also produce iNOS which is responsible for excess production of NO during inflammation. Role of $\mathrm{NO}$ in lung carcinogenesis is complex and depends on its local concentration. NO causes DNA damage, lipid peroxidation, and also the formation of nitrosamine carcinogens. It also has a regulatory role in angiogenesis, platelet aggregation, vasodilatation and metastasis [41]. NO inactivates PGI synthase, lowering the production of $\mathrm{PGI}_{2}$ that has antitumorigenic activity and acts to raise the levels of $\mathrm{PGE}_{2}$ that has the pro-tumorigenic activity [42].

We conclude that a single intratracheal dose of DMBA at $20 \mathrm{mg} / \mathrm{kg}$ of body weight induces lung cancer in rats in a time dependent manner. COX-2 is upregulated during the process and its inhibition by the preferential COX-2 inhibitor, Diclofenac leads to the prevention in the progression of lung cancer. The mechanism of action of Diclofenac seems to be mediated by the restoration of apoptosis by induction of caspase- 3 and inhibition of COX-2. Diclofenac thus proved to be a potential chemopreventive agent in lung cancer and needs to be further probed for the molecular mechanism such as signal transduction pathway in apoptosis.

\section{References}

1. Greenlee, R.T.; Murray, T.; Bolden, S.; Wingo, P.A. Cancer statistics, 2000, $C A$ Cancer J Clin.,2000,50,7-33.

2. Wynder, E.L.; Hoffmann, D. Smoking and lung cancer: scientific challenges and opportunities, Cancer Res.,1994,54,52845295.

3. Hoffmann, D.; Hoffmann, I. The changing cigarette, 1950-1995. J Toxicol Environ Health, 1997,50,307-364.

4. Li, N.; Chen, X.; Liao, J.; Yang, G.; Wang, S.; Josephson, Y.; Chi, H.; Chen, J.; Huang, M.T.; Yang, C.S. Inhibition of 7, 12dimethylbenz[a]anthracene (DMBA)-induced oral carcinogenesis in hamsters by tea and curcumin, Carcinogenesis,2003,23,1307-1313.

5. Buters, J.; Martinez, L.Q.; Schober, W.; Soballa, V.J.; Hintermair, J.; Wolff, T.; Gonzalez, F.J.; Greim, H. CYP1B1 determines susceptibility to low doses of 7, 12dimethylbenz $[a]$ anthracene induced ovarian cancers in mice: correlation of CYP1B1mediated DNA adducts with carcinogenicity, Carcinogenesis,2003,24,327-334.

6. Weimer, T.L.; Reddy, A.P.; Harttig, U.; Alexander, D.; Stamm, S.C.; Miller, M.R.; Baird, W.; Hendricks. J.; Bailey, G. Influence of beta-Naphtoflavone on 7,12Dimethybenz(a)anthracene metabolism, DNA adduction and tumorigenicity in rainbow trout, Toxicol. Sci.,2000,57,217-228.

7. Willey, J.C.; Coy, E.L.; Frampton, M.W.; Torres, A.; Apostolakos, M.J.; Hoehn, G.; Schuermann, W.H.; Thilly, W.G.; Olson, D.E.; Hammersley, J.R.; Crespi, C.L.; Utell, M.J. Quantitative RT-PCR measurement of

(C) 2010 by NWPII. All rights reserved. 
cytochromes p450 1A1, 1B1, and 2B7, microsomal epoxide hydrolase, and NADPH oxidoreductase expression in lung cells of smokers and nonsmokers, Am J Respir Cell Mol Biol.,1997,17(1),114-124.

8. Kleiner, H.E.; Vulimiri, S.V.; Reed, M.; Uberecken, A.; DiGiovanni, J. Role of cytochrome P450 1a1 and 1b1 in the metabolic activation of 7,12-dimethylbenz[a]- anthracene and the effects of naturally occurring furanocoumarins on skin tumor initiation, Chem Res Toxicol.,2002,15,226-235.

9. Hirsch, F.R.; Lippman, S.M. Advances in the biology of lung cancer chemoprevention, $J$ Clin Oncol.2005,23(14),3186-3197.

10. Ruegg, C.; Zaric, J.; Stupp, R. Nonsteriodal anti-inflammatory drugs and COX 2 inhibitors as anti cancer therapeutics: hypes, hopes and reality, Ann Med.,2003,35,476-487.

11. Wolff, .; Saukkonen, K.; Anttila, S.; Karjallainen, A.; Vainio, H.; Ristimaki, A.; Expression of cyclooxygenase-2 in human lung carcinoma, Cancer Res., 1998,58,49975001.

12. Castelao, J.E.; Bart, R.D.; DiPerna, C.A.; Sievers, E.M.; Bremner, R.M. Lung cancer and cyclooxygenase-2, Ann Thorac Surg.,2003,76,1327-35.

13. Subbaramaiah, K.; Dannenberg, A.J. Cyclooxygenase 2: a molecular target for cancer prevention and treatment, Trends Pharmacol Sci.,2003,24(2),96-102.

14. Hull, M.A. Cyclooxygenase-2: How good is it as a target for cancer chemoprevention?, Eur J Cancer,2005,41,1854-1863.

15. Wiese, F.W.; Thompson, P.A.; Kadlubar, F.F. Carcinogen substrate specificity of human COX-1 and COX-2, Carcinogenesis,2001,22,5-10.

16. Kam, P.C.A.; See, A.U.L. Cyclo-oxygenase isoenzymes: physiological and pharmacological role,

Anaesthesia,2000,55,442-449.

17. Kaur, J.; Sanyal, S.N. Association of PI3kinase and Wnt signaling in non-steroidal antiinflammatory drug-induced apoptosis in experimental colon cancer, Am. J. Biomed. Sci., 2009,1(4),395-405.
18. Saini, R.K., Sanyal, S.N. Pulmonary carcinogenesis in mice with a single intratracheal instillation of 9,10-Dimethyl Benz(a)anthracene, Drug Chem Toxicol.,2008,31,459-471.

19. Pearse, A.G.E. In: Histochemical, theoretical and applied. 3rd Edn. Vol. 1. Churchill Livingstone (London) 1968,p-660.

20. Kaur, J.; Sanyal, S.N. Oxidative stress and stress-signaling in chemoprevention of early colon cancer by diclofenac, Am. J. Biomed. Sci., 2010,2(1),63-78.

21. Sambrook, J.; Fritsch, E.F.; Maniatis, T. Molecular Cloning: a laboratory manual; 2nd ed, Cold Spring Harber Laboratory Press, Cold Spring Harber, New York. 1998, p-192.

22. Schwartz, L.M.; Osborne, B.A. Cell Death, Method Cell Biol.,1995,46,15-18.

23. Mittal, N.; Sanyal, S. intratracheal instillation of surfactant inhibits lipopolisachharideinduced acute respiratory distress syndrome in rats, Am. J. Biomed. Sci., 2010,2(2),192-201.

24. Bradford, M.M. A rapid and sensitive method for the quantitation of microgram quantities of protein utilizing the principle of protein- dye binding, Anal Biochem.,1976,72,248-254.

25. Towbin, H.; Staehelin, T.; Gordon, J. Electrophoretic transfer of proteins from polyacrylamide gels to nitrocellulose sheets: procedure and some applications, Proc Natl Acad Sci USA,1979,76,4350-4354.

26. Stuehr, D.J.; Marletta, M.A. Synthesis of nitrite and nitrate in murine macrophage cell lines, Cancer Res., 1987,47,5590-5594.

27. Boyde, T.R.C.; Rahmatullah, M. Optimization of conditions for the colorimetric determination of citrulline, using diacetyl monoxime, Anal Biochem.,1980,107,424-431.

28. Mauderly, J.L.; Jones, R.K.; Griffith, W.C.; Henderson, R.F.; Mcclellan, R.O. Diesel exhaust is a pulmonary carcinogen in rats exposed chronically by inhalation, Toxicol Sci.,1987,9,208-221.

29. Venkatesan, P.N.; Rajendran, P.; Ekambaram, G.; Sakthisekaran, D. Combination therapeutic effect of cisplatin along with Solanum trilobatum on benzo(a)pyrene induced experimental lung carcinogenesis, Nat Prod Res.,2008, 22(12),1094-106.

(c) 2010 by NWPII. All rights reserved. 
30. Xu, L.; Deng, X. Tobacco-specific Nitrosamine 4-(Methylnitrosamino)-1-(3pyridyl)-1-butanone induces phosphorylation of $\mu$ - and m-calpain in association with increased secretion, cell migration, and invasion, J Biol Chem.,2004,279(51),5368353690.

31. Kumar, M.N.V.R.; Vadhanam, M.V.; Horn, J.; Flesher, J.W.; Gupta, R.C. Formation of benzylic-DNA Adducts resulting from 7, 12Dimethylbenz $[a]$ anthracene in Vivo, Chem Res Toxicol.,2005,18, 686-691.

32. Rioux, N.; Castonguay, A. Prevention of NNK-induced lung tumorigenesis in $\mathrm{A} / \mathrm{J}$ mice by acetylsalicylic acid and NS-398, Cancer Res., 1998,58,5354-5360.

33. Bauers, A.K.; Nield, L.D.D.; Malkinson, A.M. High cyclooxygenase 1 (COX-1) and cyclooxygenase 2 (COX-2) contents in mouse lung tumors, Carcinogenesis,2000,21(4),543550 .

34. Giuliano, F.; Warner, T.D. Ex vivo assay to determine the cyclooxygenase selectivity of non-steroidal anti-inflammatory drugs, $\mathrm{Br} J$ Pharmacol.,1999,126,1824-1830.

35. Schultz, S.; Lagasse, C.A.; Odell, K.M. Cyclooxygenase 2 (COX 2) selective nonsteroidal anti-inflammatory drugs-clinical implications of cardiovascular outcomes, $J$ Air Force Pharm., 2005,12(1),8-19.

36. Grosch, S.; Maier, T.J., Schiffmann, S.; Geisslinger, G. Cyclooxygenase 2 (COX-2)- independent anticarcinogenic effects of selective COX-2 inhibitors, J Natl Cancer Inst., 2006,98(11),736-747.

37. Hofer, M.; Hoferoya, Z.; Pedorocko, P.; Mackov, N.O. Hematopoeisis stimulating and antitumor effects of repeated administration of Diclofenac in mice with transplanted fibrosarcoma cells, Physiol Res.,2002,51,629632.

38. Riedl, K.; Krysan. K.; Põld, M.; Dalwadi. H.; Vourc'h, N.H.; Dohadwala, M.; Liu, M.; Cui, X.; Figlin, R.; Mao, J.T.; Strieter, R.; Sharma, S.; Dubinett, S.M. Multifaceted roles of cyclooxygenase- 2 in lung cancer, Drug Resist Updates,2004,7(3),169-184.

39. Chang, H.C., Weng, C.F. Cyclooxygenase-2 level and culture conditions influence NS398induced apoptosis and caspase activation in lung cancer cells, Oncol Res.,2001,8,13211325.

40. Balkwill, F.; Mantovani, A. Inflammation and cancer: back to Virchow?, Lancet,2001,357,539-45.

41. Fujimoto, H.; Ando, Y.; Yamashita, T.; Terazaki, H.; Tanaka, Y.; Sasaki, M.; Suga, M.; Ando, M . Nitric oxide synthase activity in human lung cancer, Jpn J Cancer Res., 1997,88,1190-1198.

42. Malkinson, A.M. Role of inflammation in mouse lung tumorigenesis: a review, Exp Lung Res.,2005,31,57-82. 